\title{
The Research of the Possibilities and Application of the AutoCAD Software Package for Creating Electronic Versions of Textbooks for "Engineering and Computer Graphics" Course
}

\author{
Alexey L. Khoroshko \\ Moscow Aviation Institute (MAI), Volokolamskoe Highway, 4, 125993, Moscow, Russia
}

\begin{abstract}
In this paper there are examples showing that AutoCAD (computer-aided design (CAD)) was and still is a powerful tool for technical graphics. Since Autodesk is a pioneer in the market of commercial widely available CAD systems, in this company CAD principles (which can be used by relatively unprepared users), nature and volume of teams, sequence and features of their implementation were developed.

Despite the fact that today AutoCAD is only one of many packages, and its use as a training program guarantees not only high-quality training of specialists, but also the fact that the employee can easily switch to another CAD system, intuitively understanding the principles of function.

In terms of technical performance, AutoCAD is a complete tool covering the entire spectrum of functions in the field of "Engineering and Computer Graphics" course.

Keywords - CAD, education, engineering and computer graphics.
\end{abstract}

\section{Introduction}

CAD, construction, and development of technological documentation using a personal

DOI: 10.18421/TEM93-40

https://doi.org/10.18421/TEM93-40

Corresponding author: Alexey L. Khoroshko, Moscow Aviation Institute (MAI), Moscow, Russia.

Email: khoroshkoall@mail.ru

Received: 18 April 2020.

Revised: 27 June 2020.

Accepted: 15 July 2020.

Published: 28 August 2020.

(cc) BY-NC-ND (C) 2020 Alexey L. Khoroshko; published by UIKTEN. This work is licensed under the Creative Commons Attribution-NonCommercial-NoDerivs 4.0 License.

The article is published with Open Access at www.temjournal.com computer are the most important modern means of informatization of design and technological activities, and they are an important part in the information environment within modern production, science, and education, especially higher technical schools. Among these tools related to science and technology, AutoCAD and its modifications is a major figure [1], [2].

AutoCAD is a powerful tool for automating graphical work based on personal computers. It gives the user possibilities which earlier could be realized only on big and expensive computing systems: any drawing that was drawn manually can be constructed with AutoCAD now. AutoCAD is capable to perform almost any kinds of graphic works [3], [4]. Thus, there is a high speed and simplicity in creation a drawing and its updating that, in turn, allows reducing essentially time necessary for performance of these processes, in comparison with drawing manually. In this connection, the system comes into common use, in particular, for such purposes as:

- architectural and engineering drawings execution;

- interior design and space planning;

- production of process sheets and organization charts;

- drawing production for electronic, chemical, construction, engineering, and aerospace applications;

- production of topographical and sea maps;

- ship design;

- graphic and other representation of mathematical functions;

- stage decoration;

- musical score recording;

- production of technical illustrations and diagrams, trademarks and logotypes, greeting cards, etc. for other artistic and graphic works.

However, possibilities of AutoCAD are not limited to creation of static drawings. If there are 
such packages as AutoDescRenderman, 3DStudio, AutodeskAnimator, AnimatorPro, etc. intended for coloring and "animation" of drawing, it becomes effective tool for creation a film effect and demonstration of interaction of objects [5], [6].

AutoCAD allows creation not just for normal drawing. Logically connected fragments can be placed on the allocated layers or grouped in compound objects. And we consider them as a whole entity. AutoCAD "remembers" position, size, color of the constructed objects and writes down this data in an internal database for their subsequent search, analysis, and processing. AutoCAD can function with a wide range of personal computers and graphic workstations under control of various operating systems [7], [8].

\section{Autocad as a Universal Graphics Core}

Today AutoCAD is a universal system, which includes the following:

- powerful graphic editor;

- construction parameter calculation modules (mass, volume, strength, etc.);

- design documentation (DD) subsystems;

- postprocessors for manufacturing equipment.

The objects of designing CAD are the following products:

- details;

- assembly components, nodes, blocks;

- systems (of power supply, control, ventilation, etc.).

The tasks of working with CAD are:

- product design development;

- development of DD for the designed product;

- analysis of the ability to assemble nodes.

The results of designing CAD structures are:

- graphic model of a design object on an electronic media;

- drawings of the design object on electronic media and paper:

- detail drawing;

- assembly and mounting drawings;

- schemes;

- other DD (specifications, lists).

Graphic editors of modern CAD usually meet the following requirements:

- vector format of the internal representation of the graphic model in the Random Access Memory (RAM) and in file;

- complex hierarchical structure of design objects;

- creation of DD in accordance with the requirements of standards;
- redundant system of commands and instructions providing ease of entry and editing;

- setting parameters constant for the entire input and editing process;

- presence of 2D and 3D graphics;

- ability to create and use object libraries;

- ability to base objects;

- ability to scale objects;

- ability to stratification of the design object.

Coordinates in AutoCAD are stored in the internal format as double precision floating-point numbers (twelve significant figures and mantissa). Therefore, the coordinate range is rather large. For convenience, the coordinates are entered and displayed in a userfriendly form, which he can set and change. AutoCAD can function in different linear dimension systems:

- $\operatorname{architectural}\left(11 / 2^{\prime \prime}\right)$;

- fractional (1 2/5);

- decimal (1.237);

- engineering (1.34');

- scientific $(0.73 \mathrm{E}+03)$.

In addition, we can select the units of representation of angular quantities:

- degrees / minutes / seconds (0d25' '15");

- gradians $(15 \mathrm{~g}, 7)$;

- decimal degrees (23.345);

- radians $(1 \Gamma, 23)$;

- topographic units (N 45d E,0).

The user can select the accuracy of displaying linear and angular dimensions by determining the quantity of number after the comma (up to 8).

When printing out and inserting finished blocks into the current image, the size of linear dimensions matters. In AutoCAD, we can select a size from the following list:

- dimensionless;

- inches;

- micro inches;

- feet;

- miles;

- angstroms;

- nanometers;

- microns;

- millimeters;

- centimeters;

- decimeters;

- meters;

- kilometers;

- decameters;

- hectometers;

- gigameters;

- miles;

- yards;

- light years;

- parsecs. 
The complexity of the image is determined by the amount of RAM.

AutoCAD graphics has the ability to segment, that is, we can create hierarchical models of objects. The number of hierarchy levels is not limited. Segments are inserted with an arbitrary scale (may be different along $\mathrm{X}$ and $\mathrm{Y}$ axes), rotation angle, and mirror image. There is a developed system of attributes to supplement the graphic model of the object with nongraphic information. Moreover, this information can be displayed on the drawing as a test, or it may be invisible [9], [10].

The number of layers (levels) is not limited.

AutoCAD can function with 2D (two-dimensional) and three-dimensional graphics. In two-dimensional design, we can use the Cartesian and the polar coordinate system. For a three-dimensional image, we can get 2D projections, sections, and view from a given point (it is widely used in architectural design).

The number of colors is limited by computer capabilities.

Menus can change for different applications, and a user can create his own ones.

AutoCAD has a built-in LISP language for programming complex functions not previously provided for managing and creating images.

There are various snap modes that allow to precisely setting the coordinates, entering them with large errors. For example, for a given binding modes to the ends of segments, entering another coordinate near the end of the segment, it will snap to the end of the segment (it will be transferred exactly to the end of the segment). Binding modes allow avoiding inconsistencies in the image elements at any magnification of the image on the screen and to get an accurate model of the object with a simple (not accurate) input of coordinates using the graphic cursor (using the mouse).

Unlike other CAD systems, AutoCAD has almost universal polyline primitive among elementary graphic ones, whose parts can be segments and arcs, and they have different initial and final widths. The corners of the polyline can be rounded with a given radius of curvature. AutoCAD comes with a lot of fonts. It is possible to create new fonts in addition to existing ones. Any font can be displayed in various modifications (height to width ratio, angle of inclination). The standard delivered fonts contain special icons used in engineering and architectural drawings (sign of diameter, parallelism of planes, alignment, tolerances, etc.) [11], [12].

Graphics core ACIS. ACIS is an object-oriented geometric modeling package developed by Spatial Technology for use as a geometric basis in 3D modeling applications. ACIS provides an open architecture tool for wireframe, surface, and solid modeling with a common, unified data structure.
Using a physical analogy with a car manufacturer, ACIS can be seen as an engine installed in a car.

As it is known, car manufacturers usually install other components, such as transmissions, wheels, steering gear, etc. from subcontractors to speed up the production process. It is important that all components that depend on the physical connections and the characteristics of the engine presented interact well with the engine [13].

In ACIS, this is done using component technology that extends the functionality of it. Component technologies are called ACIS husks. There are a lot of these component technologies, each of them can be presented in connection with ACIS depending on the particular area of the application.

\section{Autodesk Graphics Applications}

Due to the similarity of the design processes in various industries, AutoCAD is used in, for example, machine-building, architecture, and electrical engineering. However, since each direction still has its own specifics, Autodesk produces the so-called "vertical" solutions that are based on AutoCAD, but take into account the characteristics of each industry:

AutoCAD Architecture: for architects. Automatic generation of sections and facades from plans, automatic placement of doors and windows in walls, automatically applied marks of rooms and their areas, etc.

AutoCAD Electrical: for designers of electric schemes. Automatic numbering of wires, navigation on devices, the most extensive libraries of ready components from known manufacturers and many other things.

AutoCAD Electrical is designed specifically for the design of electrical control systems. Specialized features help to reduce design time. Creating drawings is made even easier with convenient tools for cutting wires, copying and removing components and circuits, and aligning components.

AutoCAD Electrical data transfer tools make it easy to import projects created in AutoCAD or AutoCAD LT for further development. AutoCAD data is instantly converted into intuitive AutoCAD Electrical objects. So we can start implementing ideas immediately with specialized tools.

The library of programmable logic inputs/outputs controllers contains more than 3000 components from the most well-known manufacturers. We can use them to create drawings quickly while using programmable logic inputs/outputs controllers.

Flexible possibility to place multilink schemes in drawings allows reducing number of auxiliary drawings to a minimum. During the time we insert them in horizontal and vertical directions we can easily operate characteristics of constructions, 
including intervals between links, number of links, thickness of the multilink scheme, etc. The placement of auxiliary line numbers is fully automated and carried out according to the specified settings.

AutoCAD Electrical makes it easy to view and navigate between devices. Urfer command allows quickly going to the scheme of the device that has a connection with the selected one, even if it is located on another drawing of the project. Thanks to the above functions it is possible to concentrate on the creative side of designing, forgetting about routine operations.

It is possible to transfer each inserted dependent contact to the next contact group based on the part number of the relay coil. We can display the crossreference information in the drawings in almost any format and, if necessary, create a report on it.

AutoCAD Electrical allows organizing joint work of experts in machine-building design and the design of electric control systems. The created drawings can be opened and changed in other CAD of Autodesk.

Autodesk Inventor is a family of products for industrial 3D designing, including modeling, creation of tooling, and exchange of the design data. When using the technology of digital prototypes it allows creating products of higher quality in less time.

Autodesk Inventor unites in digital prototypes data AutoCAD and 3D models Inventor. Being exact 3D representation of a finished product, digital prototypes represent a convenient tool to check the form and functionality of products before creation of their prototypes.

Industrial 3D CAD Autodesk Inventor has an intuitive parametric working environment for creation of conceptual sketches and kinematic models of details and products. Means of functional designing allow concentrating attention on working characteristics, and previously on their basis to create plastic details, steel frameworks, and rotating components. Inventor reduces the amount of work users do with individual geometry objects, giving them more time to build and improve digital prototypes. Then these prototypes help to verify the functionality of products, thereby reducing the overall cost of design and manufacturing.

When designing at Inventor, it is possible to explore different conceptual ideas. A successful initial concept is the key to a successful project solution. Ideas from paper are transferred to a parametric environment and take shape as sketches and kinematic models.

Using dynamic sketch blocks, we can quickly consider possible concepts and then obtain a fully defined 3D model of the product for digital prototyping. With the help of design wizards, remaining components such as gears, fasteners, steel structures, and hydraulic systems are quickly added.

Even before we start designing parts and products, we can consider several design ideas and choose the best one. The working environment with sketches in Inventor allows creating schematic outlines of details and mechanisms on the basis of initial design ideas quickly. For an embodiment of concepts in sketches functions with regard to construction of objects and dependence serve. While the best decision is not chosen, we can keep some variants on the screen, selecting them by different colors and types of lines.

Component generators make it possible to obtain 3D models of typical mechanism components based on their functional requirements. Work is not already limited to 2D plotting and 3D modeling frameworks. Design process is accelerated thanks to that work with details, which is conducted on the basis of mechanical interrelations, instead of only descriptions of geometry (lines, arcs, circles, dependences). Details and products are created on the basis of input comprise characteristics of real objects. Inventor has component generators for threaded connections, shafts and bushings, gear work, cord, chain and screw drive, O-rings, and springs.

The plastic details designed in Autodesk Inventor, are ready to transfer in manufacture. The maximum flexibility is reached by a combination of geometrical objects Inventor with models of the external surfaces developed in Autodesk Alias Designer, Autodesk Alias Modeler, Autodesk Alias Auto Studio, etc. The associative communication between family Autodesk Alias and Inventor allows designers to pass faster to detailed designing and to finish it sooner. In process of concept completion the changed forms which are easily introduced by design divisions arrive from industrial designers. Functions of construction in conjunction with conjugations on the basis of rules and multi-body the working environment make the contribution to simplification and acceleration of designing the cast plastic details.

By Autodesk Inventor it is possible to create geometrical elements of the most difficult form by a combination of the elementary bodies and surfaces. The user completely operates with characteristics of forms, such as smoothness of interface and continuity. Construction of elements in cross-sections in various ways, by means of shift, and also creation of various kinds of conjugations between form elements is possible.

Solid-state models can be modified using surfaces: created in Inventor and imported from other systems. Using surfaces, we can add material to existing bodies and cut them off; we can also create new 3D bodies from a set of surfaces that form a closed volume. 
The product achieves an optimal balance between the aesthetic requirements of the mold connector lines and the economic efficiency of the detail production. Cast parts are described by appropriate division of $3 \mathrm{D}$ components and the location of the connector surface along silhouette curves. There are also functions that divide the detail into several parts for easy processing.

The product makes it possible to quickly add technological plastic elements for the parts to be transferred to production. Due to the integrated recording of connector direction and casting slope, Inventor is able to form multi-element plastic components in one operation. So, typical components that do not require sketching such as gratings, bosses, flanges, and stops are created.

While designing the details from sheet material Inventor provides productivity growth, giving a digital prototype where the information for manufacturers (parameters of downcut tools, tables of bending, etc.), 3D model of a finished part, and environment for editing a sweep with which engineers within industrial departments can optimize a detail are connected.

Pattern unfolding can be controlled with styles that parameters define (material thickness, bending rules, and angular die-cutting). Inventor can use linear deployments, user-defined rules, and custom bending tables when receive deployments.

It is possible to use bent profiles in digital prototypes. The contour rolling function makes it easy to create profiles; there is the support for addition structural elements to parts and the creation of their sweeps.

Parameter rows and product families can be developed using configurations that exclude or replace individual components, changing dimensions and assembly dependencies. An automatically generated parametric row table can be placed in the drawing of this product.

The frame generator is used to design structures (frames) from standard profiles. The ends of the profiles are cut for welding. Users can create their own profiles and add them to the library.

The program ensures high quality of welded structures and their documentation. Simulation of welded seams in 3D environment really represents processes of cutting, welding, and post-processing. It is possible to calculate the welded structure and weld roll volume. In addition, we can automatically create 3D models of welds, associatively display and designate them in drawings according to given standards.

The use of library components allows achieving greater standardization in the enterprise. Such components are not difficult at all to create and then repeatedly apply in projects. For components search there is a browser with convenient functions of search and filtering of elements. The library contains more than 650000 model elements. These are nuts, bolts, screws and other details. We can create new files in the library and place our own models there.

Raster Design vectoring software enables architects, engineers, engineering systems and industrial designers to reduce drawing time by using scanned drawings and plans obtained with AutoCAD software products. Key features include the following:

- image show;

- editing and image clean up;

- working with raster objects;

- vectorization tools, including optical character recognition (OCR).

Possibilities to display the images provided in the program for work with raster graphics AutoCAD Raster Design, allow to lower expenses of time essentially. The presented functionality helps users to choose from a wide spectrum of the given images necessary ones, which will be used in projects.

The program of work with raster graphics AutoCAD Raster Design possesses powerful possibilities of editing and image clean up. It allows architects, engineers, engineering systems designers, and industrial designers to lower expenses of time for drawing by use of the scanned drawings and the plans received by means of software products on platform AutoCAD.

With powerful image clean up tools, it become clear, understandable, and easy to use. The Despeckle command cleans images from random pixels resulting from fuzzy patterns and photocopies. With the Bias command, distortion of proportions can be corrected. The Mirror command flips the image along a horizontal or vertical axis, changing incorrectly scanned drawings.

It is possible to significantly reduce the time required to clean scanned drawings and maps with the Touchup tool, which is similar to the drawing tool in Microsoft Paint. Using the hand-drawing feature with many different brush sizes, we can color the foreground and background.

We can enhance the clarity of scanned images by overlaying shadows without reducing brightness. We can improve the quality of scanned photos and satellite images with gamma correction and a linear or fragment curve.

OCR applied to drawings that contain text, and other similar features reduce drawing time by using scanned drawings and plans obtained with AutoCAD software.

Eliminating time-consuming manual input saves time and increases accuracy when converting drawings with text inscriptions. Handwritten and 
printed texts and tables are recognized in raster images. Based on the recognized image, we create single-line and multi-line AutoCAD text. We can check if the recognition results are correct with the dictionary.

AutoCAD Raster Design, using with AutoCAD Map 3D, provides designers of engineering structures, transportation networks, and master plan, as well as cartographers and GIS specialists with the ability to edit raster images, visual analysis, and processing the cartographic images.
When used with AutoCAD Civil 3D, AutoCAD Map 3D, and Autodesk Topobase ${ }^{\mathrm{TM}}$, AutoCAD Raster Design provides the tools to display and analyze georeferenced images. The ability to acquire and use geographic data from a variety of sources enables us to easily and efficiently edit raster images, visually analyze, and process spatial images.

The program allows using images provided by central and local government agencies over the Internet or on CDs in common Lizard Tech ECW and MrSID formats. We can insert Digital Globe Quick Bird satellite images, Landsat-FAST multispectral images and National Imagery Transmission Format (NITF) images into the projects.

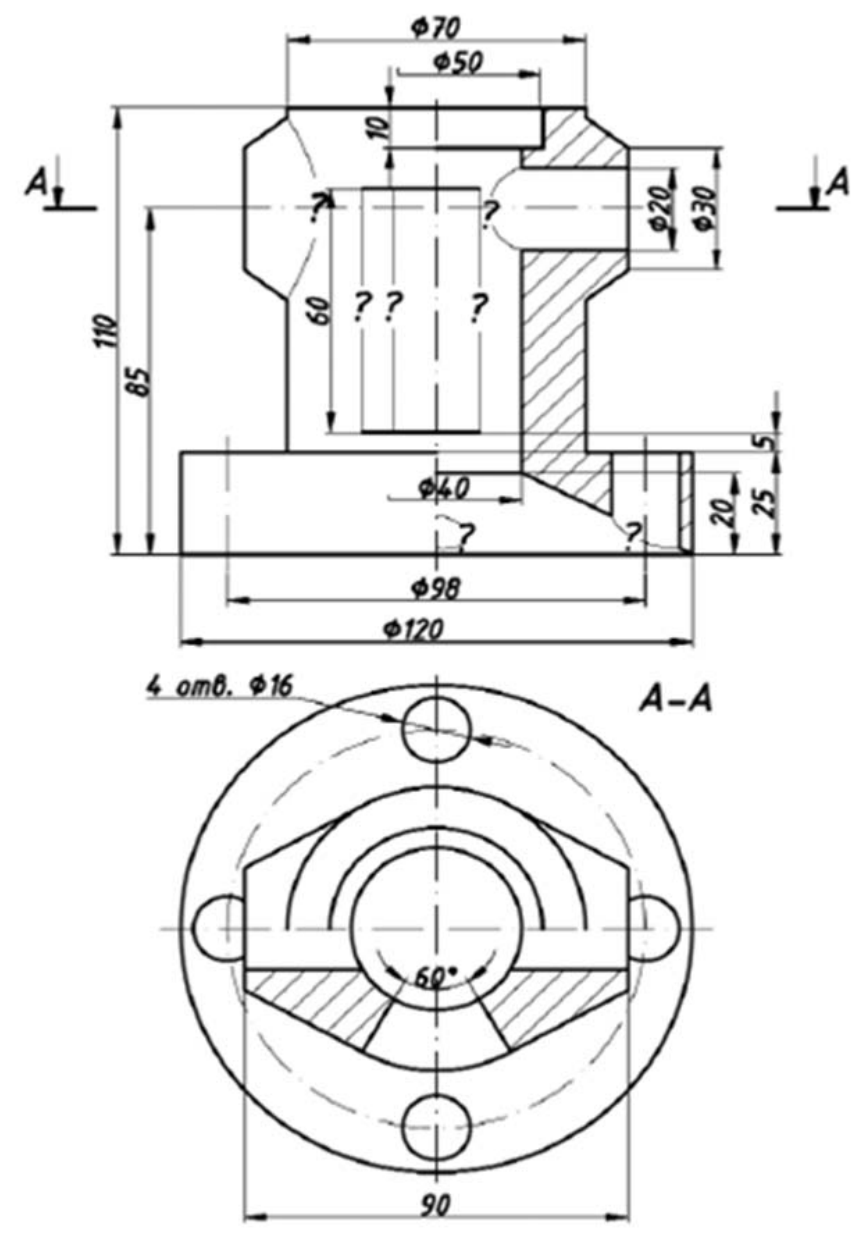

Figure 1. Task example

4. Applying Autocad for Training in "Engineering and Computer Graphics" Course on the Example of Drawing a Pattern of "Body Frame" Type

1. Solution preparation:

- holding the axes of projections, dividing the drawing field into four equal parts; carrying out bisector by means of a polar method of assignment in relative coordinates;
- holding a symmetry axis for the three species, dividing in half the quarter of the drawing field;

- using Endpoint, Midpoint, Center and Intersection snap-ins building a circle of cylinder bases in the top view, and then the other projections in the front and on the left view, using the Offset tool;

- drawing a centerline of the truncated cone in the upper part of the pattern, and build its 
projections in three views using the Offset tool and Tangent anchorage (for the top view);

- building a circle on top, on which the centers of cylindrical holes on the base of the pattern are located, and creation of holes using the Array tool in polar mode (Figure 2.).
2. Construction of intersection lines for surfaces:

- determining the points of intersection of the truncated cone with the cylinder using the method of auxiliary sectional planes (frontal projection), building these points on the left side of the front view and on the left side of the view;

after drawing the necessary number of points, we connect them with a smooth curve using the Spline tool and then remove the auxiliary lines (Figure 2.).

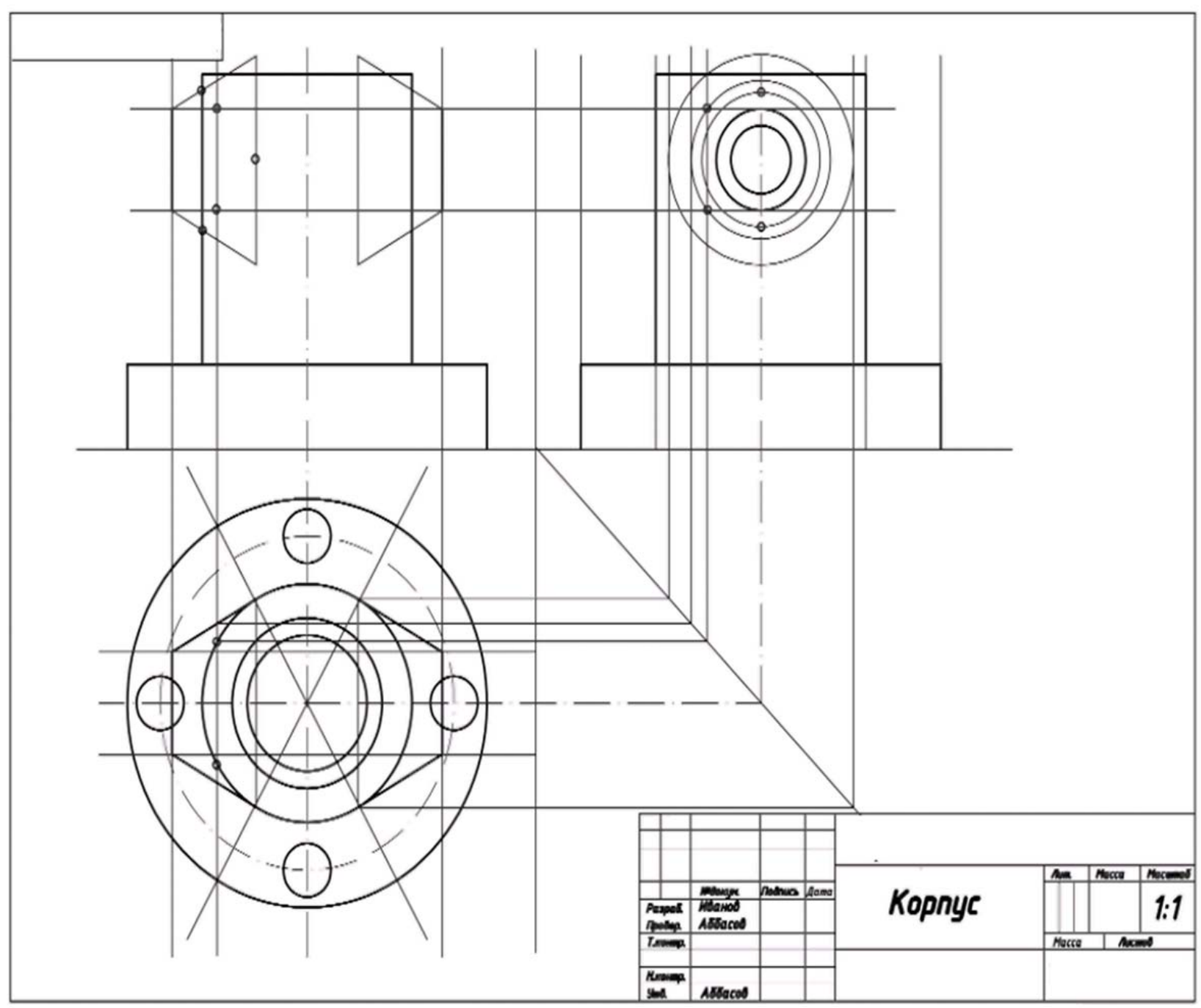

Figure 2. Construction of the third type and definition of intersection lines

3. Construction of the cut in the front and top views:

- building the slit on the right side of the front view;

- determining the reference point for intersection of a cylindrical bore inside a truncated cone in the front form with a through cylindrical bore inside the part;

- determining the reference point for the intersection of the cylindrical bore at the base of the part with the inner bore as a truncated cone in the front view;

- determining the boundary points of the projection of the cylindrical bore (along the centerline) in the inner part of the base;

- connection regarding the intersection points of the smooth curve (in three places where there were question marks) using the Spline tool, delete auxiliary lines (Figure 3.). 


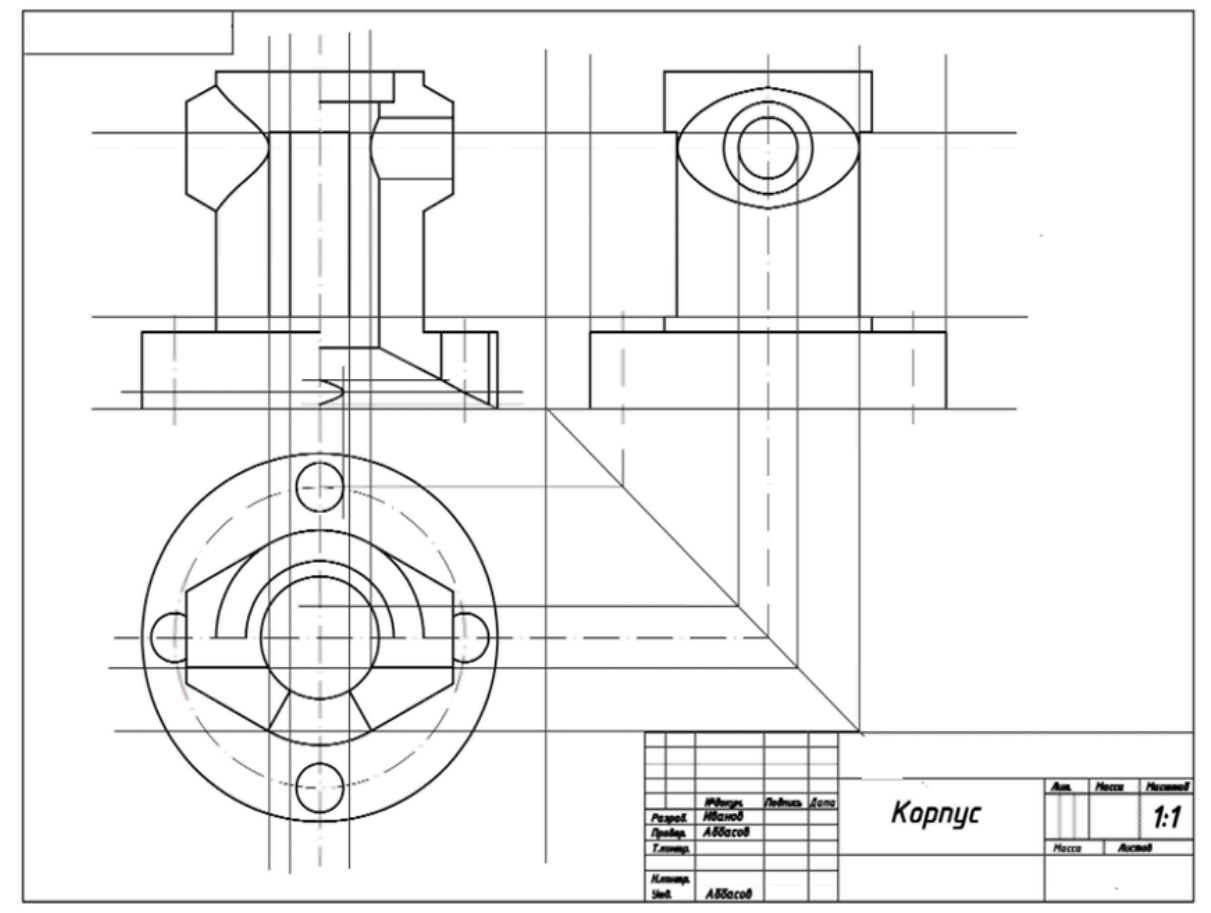

Figure 3. Construction of intersection lines

4. Construction of a specified horizontal section in the top view and a through prismatic hole in all images:

- construction of a horizontal cut from the top on a sectional plane A-A;

- construction, according to the given sizes, of projections through prismatic aperture in the views at the front and left;

- deinstalling the auxiliary constructs;
- using the Hatch tool to shade cross-sectional shapes in images, checking that they are closed;

- indicating the position of the sectional plane A-A and marking the cut (Text layer).

- using the Dimension Style tool to set parameters;

- dimension with the Dimension tool;

- saving the drawing and printing it out (Figure 4.).

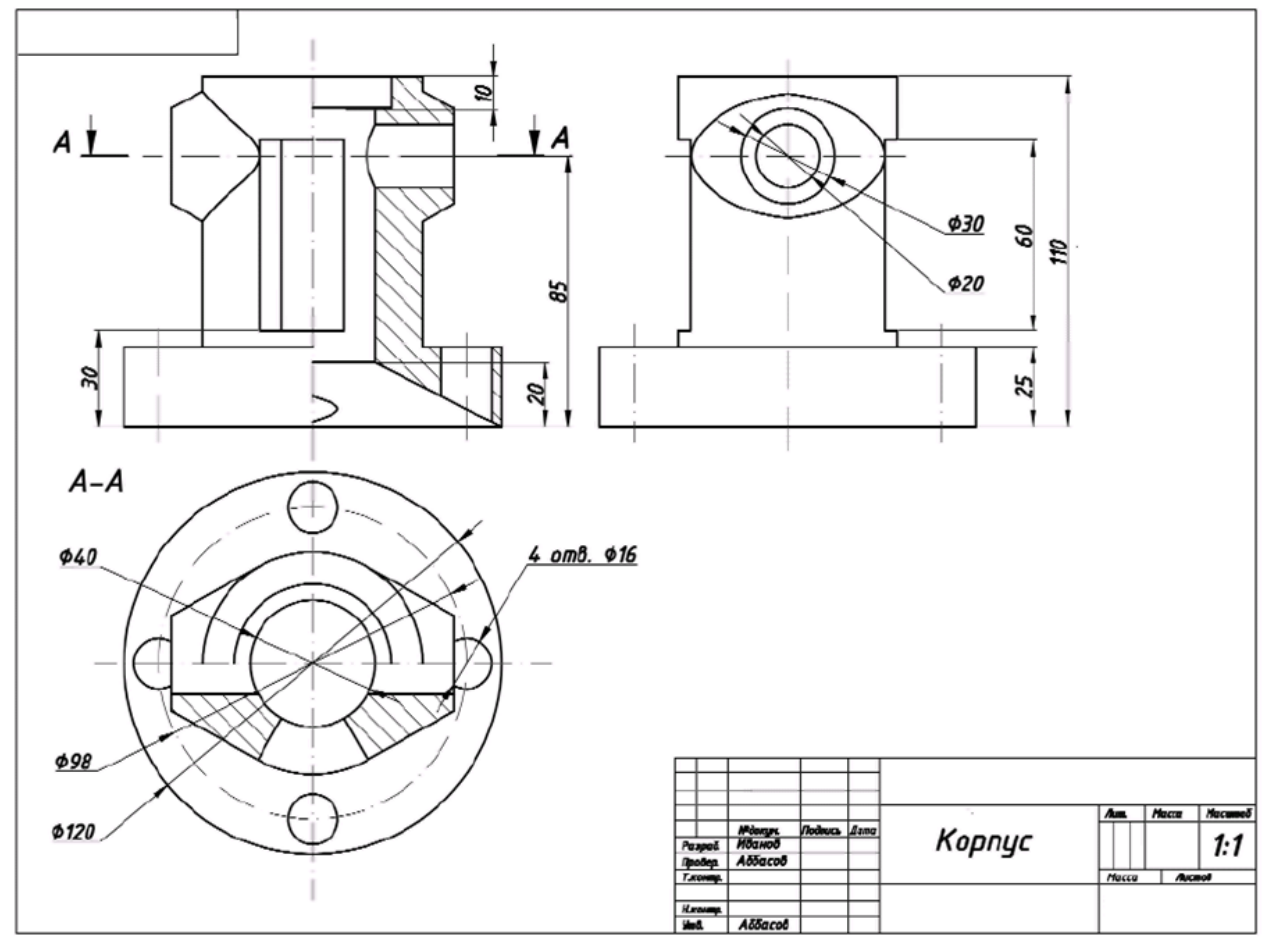

Figure 4. Finished drawing of the "Korpus" type part 


\section{Conclusions}

The considered examples show that AutoCAD was and remains powerful means of a technical drawing.

In this regard, the system has the widest application and is used for the following purposes:

- architectural and engineering drawings execution;

- interior design and space planning;

- production of process sheets and organization charts;

- drawing production for electronic, chemical, construction, engineering, and aerospace applications;

- production of topographical and sea maps;

- ship design;

- graphic and other representation of mathematical functions;

- stage decoration;

- musical score recording;

- production of technical illustrations and diagrams, trademarks and logotypes, greeting cards, as well as for other artistic and graphic works.

- in terms of technical execution, AutoCAD is a full-fledged tool that covers the entire range of work in the discipline of "Engineering and Computer Graphics".

\section{References}

[1].Grechka, M. (2008). ElectriCS + Autodesk Inventor: steps to integrated automation. CAD and graphics, 11, $16-18$

[2].Finkelstein, E. (2009). AutoCAD 2010 and AutoCAD LT 2010 Bible (Vol. 572). John Wiley \& Sons.

[3].Nikulin, E. A. (2005). Komp'yuternaya geometriya i algoritmy mashinnoi grafiki (Computer Geometry and Algo rithms of Computer Graphics), St. Petersburg: BKhV Peterburg.

[4].Patpatiya, Parth and Sharma, Shailly and Bhatnagar, Varun and Tomar, Jyoti and Shalu, Jyoti Kumari, Approaches for Concising AutoCAD Files (September 2, 2019). Proceedings of International Conference on Advancements in Computing \& Management (ICACM) 2019.

[5]. Norenkov, I. P. (2005). Kratkaja istorija vyčislitel'noj tehniki. Informacionnye tehnologii, (9), 2-32.

[6]. Shumaker, T. M., Madsen, D. A., Madsen, D. P., Laurich, J. A., Malitzke, J. C., \& Black, C. P. (2013). AutoCAD and Its Applications Comprehensive 2014. Goodheart-Willcox Co..

[7]. Abbasov, I. B. (2007). Create computer drawings in AutoCAD 2007/2008. Moscow: DMK Press.

[8].Ozkaya, S. I. (2018). FRACOR-software toolbox for deterministic mapping of fracture corridors in oil fields on AutoCAD platform. Computers \& Geosciences, 112, 9-22.

[9].Bondrea, M. V., Naş, S., Sestraş, P., Cornel, A., \& Fărcaş, R. (2017). Achieving Basic and Cadastral Database Related to a Block In The Built Sandulesti Commune, Cluj County, by using Software Gis Autocad Map. International Multidisciplinary Scientific GeoConference: SGEM, 17, 43-50.

[10]. Safiee, Z., Hanapi, Z., \& Sheh, Y. S. (2019). Factors Affecting the Readiness of Using AutoCAD Software in Teaching. International Journal of Academic Research in Business and Social Sciences, 9(5), 128137.

[11]. Khoroshko, L. L., Ukhov, P. A., \& leonidovich Khoroshko, A. (2018). The Use CAD/CAE Systems to Create E-Learning Courses on Technical Subjects at University. International Journal of Engineering Pedagogy (iJEP), 8(2), 64-71.

[12]. Liu, S. (2017). 3D modeling of Complex Structure based on AutoCAD VBA. International Journal of Engineering Research, 6(11), 472-474.

[13]. Khoroshko, L. L., \& Khoroshko, A. L. (2016). The use of parametric CAD in the educational process. VOENMEKH. Bulletin of the Baltic State Technical University, 31, 58. 